\title{
El turismo comunitario como herramienta de desarrollo de los pueblos indígenas: interrogantes en el caso de los shuar del Alto Nangaritza (Ecuador)*
}

\author{
MARÍA JESÚS BERLANGA ADELL ${ }^{* *}$ \\ MARITZA ELIZABETH OCHOA OCHOA ${ }^{* *}$ \\ Artículo de investigación científica sobre turismo comunitario y desarrollo de poblaciones \\ indígenas.
}

Recibido: 9 de diciembre del 2019 - Evaluado: 27 de abril del 2020 - Aceptado: 7 de mayo del 2020

Citar como: Berlanga Adell, M. J. y Ochoa Ochoa M. E. (2020). El turismo comunitario como herramienta de desarrollo de los pueblos indígenas: interrogantes en el caso de los shuar del Alto Nangaritza (Ecuador). Hallazgos, 17(34), 55-78. Dor: https://doi.org/10.15332/2422409X.5450

Artículo derivado del proyecto de investigación "El desarrollo del turismo comunitario en las comunidades shuar del Alto Nangaritza. Un proyecto para el fortalecimiento de las capacidades locales de paz", financiado por el Programa Prometeo de la Secretaría Nacional de Ciencia y Tecnología (Senescyt) del Gobierno de Ecuador.

* Doctora en Cooperación al Desarrollo, Universidad Católica de Valencia, Instituto Universitario de Antropología, Valencia (España).

Correo electrónico: m.jesus.berlanga@uv.es ORCID: 0000-0001-8805-9127

*** Licenciada en Derecho, Universidad Técnica Particular de Loja, Departamento de Ciencias Jurídicas, Loja (Ecuador).

Correo electrónico: meochoa@utpl.edu.ec

ORCID: 0000-0002-7196-9914 


\section{Resumen}

En este artículo analizamos el turismo comunitario como potencial instrumento de desarrollo para las poblaciones locales. Desde los años ochenta, algunos grupos indígenas de Ecuador han puesto en marcha diversas iniciativas de turismo comunitario. Tras la realización de un prolongado trabajo de campo antropológico en la zona del Alto Nangaritza (Ecuador), nuestro objetivo es mostrar las contradicciones que el turismo comunitario supone para las poblaciones locales, en concreto para las comunidades shuar de la región. Frente a un turismo que pretende empoderar a las comunidades, al desarrollarse en entornos sociales cada vez más desestructurados, esta actividad termina potenciando un individualismo que deteriora progresivamente los valores comunitarios. Esta experiencia nos muestra cómo el turismo comunitario requiere el fortalecimiento previo de las estructuras comunitarias y de la puesta en marcha de un sistema organizativo de control colectivo de los beneficios derivados de esta actividad.

Palabras clave: comunidades indígenas, Ecuador, impactos, turismo comunitario. 


\title{
Community-based tourism as a development tool for indigenous peoples: questions in the case of the Shuar of Alto Nangaritza (Ecuador)
}

\begin{abstract}
In this article we analyze community-based tourism as a potential development instrument for local populations. Since the 1980s, some indigenous groups in Ecuador have launched various community-based tourism initiatives. After making a lengthy anthropological fieldwork in the area Alto Nangaritza (Ecuador), our objective is to show the contradictions that community-based tourism represents for local populations, specifically for the Shuar communities in the region. Faced with a tourism that aims to empower communities, by developing in increasingly unstructured social environments, this activity ends up promoting an individualism that progressively deteriorates community values. This experience shows us how community-based tourism requires the prior strengthening of community structures and the implementation of an organizational system of collective control of the benefits derived from this activity.
\end{abstract}

Keywords: indigenous communities, Ecuador, impacts, community-based tourism.

\section{O turismo comunitário como ferramenta de desenvolvimento dos povos indígenas: interrogantes no caso dos shuar do Alto Nangaritza (Equador) \\ Resumo}

Neste artigo, analisamos o turismo comunitário como potencial instrumento de desenvolvimento para as populações locais. Desde 1980, alguns grupos indígenas do Equador vêm colocando em funcionamento iniciativas de turismo comunitário. Após a realização de um prolongado trabalho de campo antropológico na área do Alto Nangaritza (Equador), nosso objetivo é mostrar as contradições que o turismo comunitário revela para as populações locais, em específico para as comunidades shuar da região. Diante de um turismo que pretende empoderar as comunidades ao ser desenvolvido em entornos sociais cada vez mais estruturados, essa atividade acaba potencializado um individualismo que deteriora progressivamente os valores comunitários. Essa experiência evidencia como o turismo comunitário requer o fortalecimento prévio das estruturas comunitárias e do funcionamento de um sistema organizacional de controle coletivo dos benefícios derivados dessa atividade.

Palavras-chave: comunidades indígenas, Equador, impactos, turismo comunitário. 


\section{Introducción}

Desde los años ochenta son ya numerosas las iniciativas de turismo comunitario (TC) puestas en marcha en Ecuador por distintos actores, algo que ha venido ocurriendo en gran parte de los países en desarrollo. El TC se ha presentado, tanto en Ecuador como en otros países, como una potencial herramienta para el desarrollo de las comunidades locales, a partir de la comprensión del desarrollo como un proceso que va más allá del crecimiento económico y que constituye un proceso global y multidimensional (Unesco, 1994, p. 25). Desde esta perspectiva, el TC se ha visto como una forma de empoderamiento de las comunidades, al ser ellas mismas las que se encontrarían en la base de esta actividad, lo cual presupone una gestión comunitaria y unos beneficios colectivos (García-Andreu y Ullán, 2019). Son estas características las que han despertado el creciente interés de las ONG y de otro tipo de entidades de desarrollo por impulsar el TC, donde Ecuador es uno de los países que ha alcanzado un mayor desarrollo (García, 2016).

No obstante, la implementación del Tc adquiere una notable complejidad, en tanto que habitualmente se desarrolla en entornos donde son frecuentes los conflictos alrededor de la gestión de los recursos económicos y naturales, así como de la gestión sobre la propia identidad o los conflictos relativos a las relaciones de género y parentesco dentro de las comunidades. Además, el Tc viene siendo protagonizado frecuentemente por grupos indígenas o minoritarios, con un fuerte componente étnico, que se enfrentan al racismo y la desvalorización por parte de la sociedad dominante. En este marco, el TC suele presentarse, sobre todo, a los grupos indígenas como una oportunidad que les permitirá un mayor control sobre sus recursos y su "progreso".

El caso de los shuar, que habitan en el Alto Nangaritza (provincia de Zamora, Ecuador), y que centra el interés de este artículo, es un buen ejemplo de este tipo de dinámicas. Como veremos, en el territorio shuar el proceso de desarrollo ha sido determinado, primero, por la influencia de los misioneros y, más tarde, por las políticas estatales, lo que ha derivado en una pérdida de control de la población sobre su propio destino. El resultado es que un pueblo antaño nómada, dedicado a la caza y la recolección, se ha visto obligado a sedentarizarse ante la colonización y la expropiación de buena parte de sus tierras, por lo que ha debido adaptar su modo de vida a la nueva coyuntura sin estar suficientemente preparado para ello. De hecho, la progresiva pérdida de control de los indígenas sobre los territorios que habitan se manifiesta aún hoy en Ecuador, cuando se siguen poniendo en marcha actividades 
de explotación de los recursos pertenecientes a comunidades indígenas sin realizar una consulta previa a la población — tal como estipula la Constitución de 2008 en su artículo 57-, lo que muestra que se ha procedido a una expropiación forzosa de sus tierras.

Al mismo tiempo, los shuar del Alto Nangaritza han mostrado su interés por el TC; consideran esta actividad como una alternativa económica que les puede ayudar a generar ingresos, más allá de la ganadería y una precaria actividad agrícola, a la vez que ejercer un mayor control y preservación del territorio, con lo que se puede tratar de frenar el avance de empresas mineras y madereras que están operando en territorios colindantes.

El principal objetivo de este artículo es mostrar las contradicciones y tensiones que el TC, visto en principio como una estrategia positiva de desarrollo, entraña para las poblaciones locales en términos identitarios y de estructura social. Nuestra hipótesis es que la implementación del Tc de modo mimético, sin atender a la complejidad del medio y la organización comunitaria local, también puede convertirse en un factor de conflictividad y desestructuración. Con este fin, iniciamos un proceso de investigación que se ha prolongado a lo largo de varios años, y que a continuación detallaremos.

Así, tras exponer primeramente la metodología que hemos empleado para llevar a cabo nuestra investigación, los siguientes dos apartados se centran en el proceso que ha seguido el Tc en Ecuador y en los efectos que este tipo de turismo ha generado. El quinto apartado profundiza en la caracterización del grupo étnico shuar como ejercicio de contextualización previo al análisis. En los apartados seis y siete nos centramos en el análisis de una primera experiencia fracasada de TC desarrollada en su territorio durante los años noventa y de una nueva iniciativa de TC que trata de ponerse en marcha en la actualidad. Finalmente, ofrecemos una serie de conclusiones y reflexiones en torno al potencial del TC.

\section{Metodología}

Las informaciones sobre las que se construye este artículo provienen fundamentalmente del trabajo antropológico y formativo llevado a cabo desde 2012 entre las comunidades shuar del Alto Nangaritza, por iniciativa del Observatorio de Conflictos Socioambientales de la Universidad Técnica Particular de Loja (UTPL), Ecuador. Desde esa fecha, la UTPL ha venido realizando una serie de talleres formativos sobre el terreno que han permitido mantener una relación constante con las comunidades 
shuar de la zona (los talleres, más de una docena, han permitido un contacto continuado con las comunidades entre 2012 y 2016, combinado con un periodo de trabajo de campo intensivo desarrollado durante seis meses en $2015^{1}$ ). Dichos talleres se iniciaron con el objetivo de analizar conjuntamente los problemas de desarrollo y los conflictos socioambientales a los que se enfrenta la población local, pero se convirtieron también en un ejercicio de observación participante sobre las mismas comunidades. En el marco de esta acción las propias comunidades plantearon el interés en implementar un proyecto de TC.

Con este objetivo se inició un diagnóstico comunitario participativo como paso previo a la formulación del proyecto que incluyó, además de numerosas reuniones con las comunidades - entre estas la asistencia a algunas de sus Asambleas Comunitarias $^{2}$ - , más de una veintena de entrevistas con los líderes comunitarios y otros actores clave. Dichas entrevistas incluyen a habitantes de las comunidades de Shaime y Miazi, tanto agentes turísticos como población no involucrada en esta actividad, responsables políticos provinciales y locales, miembros de la asociación Tayunts - formada por personas de etnia shuar de Nangaritza - y de la Fepnash Federación Provincial de la Nacionalidad Shuar en Zamora, que agrupa a las distintas asociaciones shuar de cada cantón-, representantes del Ministerio de Turismo, el presidente de la Federación Plurinacional del Turismo Comunitario del Ecuador (Feptce), representantes de ONG que vienen interviniendo en la zona, el responsable de la operadora de Tc Saraguro Rikuy, técnicos del Departamento de Turismo del Gobierno Provincial de Zamora, el presidente de la Federación Plurinacional de TC del Ecuador, así como a ecuatorianos no-shuar que poseen negocios turísticos en la región de Nangaritza. Toda esta información de carácter cualitativo se complementa con la consulta de datos estadísticos y de fuentes bibliográficas y documentales.

\section{El turismo comunitario y su surgimiento en Ecuador}

El TC ha adquirido desde hace tiempo una notable presencia mundial, particularmente en los países en desarrollo (Brohman, 1996). En 2001, World Wide Foundation

1 Para ello se contó con la financiación de una beca Prometeo de la Secretaría Nacional de Ciencia y Tecnología (Senescyt) del Gobierno de Ecuador.

2 Reuniones en las que pueden participar todas las personas adultas de las comunidades shuar, donde se debaten los problemas que les afectan y se toman decisiones de forma asamblearia. 
(WWF) trató de definir el término turismo de base comunitaria frente a otros modelos más vinculados con la protección del medio ambiente, como el turismo sostenible o el ecoturismo, poniendo ahora hincapié en las comunidades locales. Para WWF (2001), en esta nueva modalidad de turismo

[...] la comunidad local tiene un control sustancial y participa en su desarrollo y gestión, y una proporción importante de los beneficios permanece dentro de la comunidad, aceptando que el concepto de comunidad depende de las estructuras sociales e institucionales locales y que también debe abarcar iniciativas individuales dentro de la comunidad. (p. 2)

A esto se añade que el turismo de base comunitaria o TC "proporciona una forma de vida más sostenible para las comunidades locales, alienta a las comunidades mismas a participar más directamente en la conservación y genera más voluntad y beneficio local en medidas de conservación como las áreas protegidas” (p. 4).

De modo que el TC se diferencia de otras formas de turismo convencionales por el hecho principal de que las comunidades mantienen un control efectivo del negocio turístico, pasando una parte de los beneficios de este a ser gestionados colectivamente (Maldonado, 2005, p. 5). Se sobreentiende que las comunidades son las que deciden su participación en las iniciativas turísticas, a través de sus órganos de gobierno, pero estas no pretenden reemplazar las actividades económicas preexistentes en las comunidades, sino incorporarse de modo armonioso a estas, fortaleciendo la cadena productiva local (Cabanilla, 2014, p. 39). Como sostienen Ruiz y Solís (2017), "el elemento definitorio del turismo comunitario no es tanto su objeto como actividad turística, sino la forma de organizar esa actividad" (p. 13). No obstante, pese a su creciente extensión y aceptación, el Tc también ha sido objeto de importantes críticas, en especial en cuanto a la limitada participación de las comunidades en su gestión y beneficios (Tosun, 2000; Blackstock, 2005).

En Ecuador, el Ministerio de Turismo (2010) conceptualizó el TC como un modelo de gestión turística, "caracterizado por la activa participación comunitaria en la planificación y ejecución de acciones conducentes a potenciar el desarrollo sostenible de la población, mediante la reinversión de los beneficios derivados de la actividad turística”. Pero antes de su conceptualización institucional, el tc ya venía existiendo en Ecuador desde comienzos de los años ochenta (Ruiz y Solís, 2007). A juicio de Cabanilla (2014, p. 33), habría surgido de forma espontánea en respuesta a la sensación de rechazo que padecían buena parte de las comunidades rurales. También surgió con el fin de lograr un desarrollo integral, en el que fuera compatible el 
desarrollo socioeconómico con la sostenibilidad ambiental, y así reducir los desequilibrios territoriales. Para Solís (2007, pp. 23-32). Su aparición se convirtió en una estrategia de las comunidades para limitar la actividad de las empresas petroleras que en los años setenta comenzaron a actuar en sus territorios, sin respeto al medio ni a los habitantes, con la consiguiente contaminación de sus recursos y la apropiación de sus mejores tierras de cultivo. Al mismo tiempo, las poblaciones locales veían disminuidas sus posibilidades de caza y contemplaban la privatización de los recursos comunales.

De esta manera, en los años ochenta apareció el $8 \%$ de las iniciativas de TC, aunque fue en los noventa cuando este modelo turístico experimentó un importante repunte, pues tuvo el $69 \%$ de las experiencias, mientras que en 2001 y 2002 apareció otro $19 \%$ (Cabanilla, 2014, pp. 33-39). Pese al tiempo que llevaban poniéndose en marcha iniciativas de TC, no fue sino hasta 2002 que se consideró oficialmente esta modalidad turística en la nueva Ley de Turismo, que le permitió adquirir plena forma jurídica. En ese año, el Estado reconoció el TC y sus organizaciones, y con esto ubicó el TC al mismo nivel que el resto de modalidades turísticas. Pero fue a partir de 2005 cuando se dio una verdadera consolidación del TC en Ecuador (Solís, 2007, p. 27), de tal modo que en el Plan de Desarrollo Nacional (2008-2013) se incorporó como objetivo del Estado el fomento del TC (Cabanilla, 2014, p. 33).

\section{Los efectos potenciales del turismo comunitario}

La mayor parte de los proyectos de TC que se dan en Ecuador se ubican en el territorio amazónico del país. En la Amazonía, en 2006, se dieron 21 iniciativas de Tc, lo que afectó a grupos étnicos como los kichwua, huaoraní, shuar, shiwiar o záparo (Feptce, 2007). En 2008 ya había cerca de 60 comunidades indígenas que ofrecían Tc (Ruiz et ál., 2008).

En este sentido, hay que tener en cuenta la fragilidad y el alto valor ecológico de este territorio amazónico. Tal como expresa Guerrero (2008, p. 34), a través del TC muchas comunidades buscan preservar y fortalecer zonas de especial valor ecológico, y como alternativa económica a otras actividades mucho más agresivas con el medio ambiente. Así, Solís (2007, p. 27) explica que un primer gran eje para 
el desarrollo del TC fue la resistencia de las comunidades indígenas a la presencia de empresas madereras, petroleras y de otras actividades productivas-extractivas que desde los años setenta en adelante aparecieron en sus territorios, y tuvieron un fuerte impacto para el medio ambiente, el tejido social y las condiciones de vida de los pueblos indígenas.

Pero si hablamos de los beneficios que conlleva el Tc, también hemos de hacer mención a sus aspectos positivos sobre las culturas indígenas. Esta modalidad turística se comenzó a desarrollar en los años ochenta y noventa, momento en que se produjo una creciente toma de conciencia en el mundo sobre la importancia de los pueblos indígenas (Solís, 2007, p. 32). Como resultado, muchas comunidades indígenas conciben el TC como una herramienta que puede fortalecer y recuperar elementos culturales en los que se basa su identidad étnica (Guerrero, 2008, p. 34; Fetpce, 2007).

Así mismo, el Tc ha sido acogido por las comunidades gracias a su potencialidad en la mejora del bienestar, puesto que les permite a sus habitantes mantenerse en el territorio, mejorar su alimentación y sus viviendas, incrementar su autoestima, mejorar la capacitación de la población y empoderar a las mujeres (Guerrero, 2008, p. 34; Timothy, 2002, p. 149). El TC se presenta, pues, como un factor multiplicador del cambio en un contexto en el que se trata de escapar de la pobreza, algo fundamental en un territorio (el amazónico) en el que se concentran los mayores niveles de pobreza del país (Chiriboga y Wallis, 2010, p. 5), y donde en el $90 \%$ de los hogares pobres hay población indígena (Solís, 2007, p. 33).

\section{Las comunidades shuar del Alto Nangaritza}

Los shuar son un grupo étnico que habita fundamentalmente en la región amazónica de Ecuador. Nuestra investigación se centra en las comunidades shuar que habitan en la región del Alto Nangaritza (provincia de Zamora Chinchipe), región representada en las figuras 1 y 2 . En ella viven 1371 personas de la etnia shuar, entre una población total de 5196 habitantes (Instituto Nacional de Estadística y Censos [INEC], censo 2010). Esta es una región de gran biodiversidad y alto valor ecológico. 


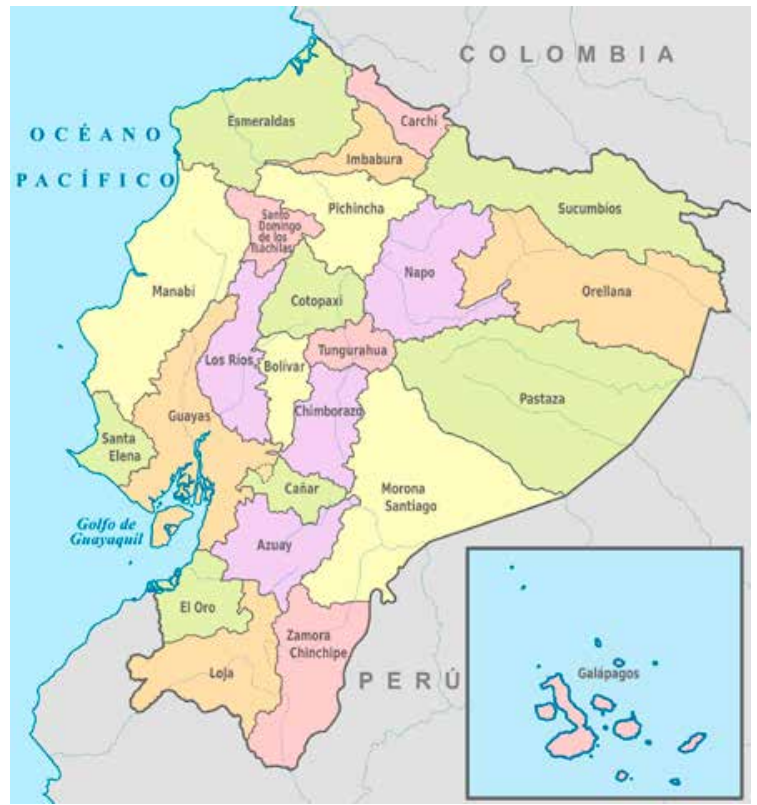

Figura 1. Localización de la provincia de Zamora Chinchipe en el mapa de Ecuador Fuente: Wikimedia Commons (2019a).

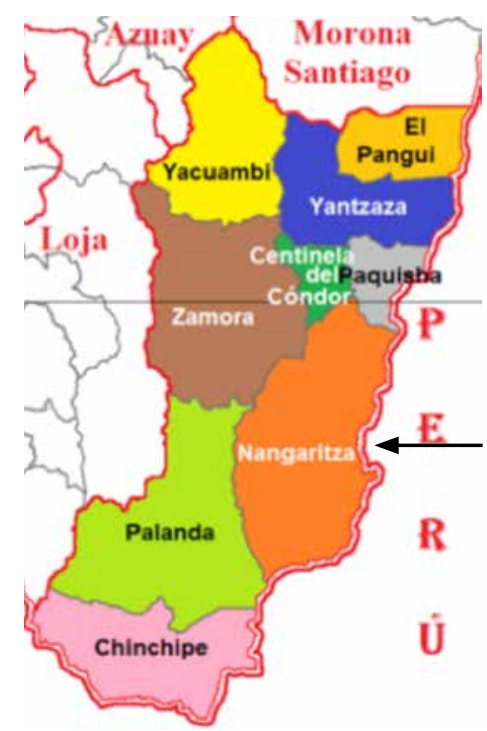

Figura 2. Localización del cantón de Nangaritza en la provincia de Zamora Chinchipe Fuente: Wikimedia Commons (2019b). 
En el Alto Nangaritza existen en la actualidad diez comunidades, de las que siete están habitadas enteramente por población shuar. En dos de ellas, Shaime y Miazi, centramos nuestro análisis. Las poblaciones shuar de Nangaritza mantuvieron hasta hace pocas décadas una economía de subsistencia, en la que se practicaba el nomadismo. Sin embargo, en la actualidad se han visto obligados a sedentarizarse, debido a la escasez de territorio disponible a causa de la colonización de tierras por poblaciones llegadas desde otras provincias, que durante décadas ha sido animada por el Estado. Esta situación los ha obligado a mantener un nuevo modo de vida para el que no estaban suficientemente preparados, y los ha impulsado a practicar nuevas actividades económicas, como la ganadería vacuna, una débil agricultura orientada a su comercialización, la explotación de recursos madereros, un incipiente turismo, la minería artesanal, la participación esporádica como asalariados en empresas mineras que operan en la zona o la emigración a las ciudades cercanas donde trabajan como asalariados. Todo ello ha supuesto un cambio de mentalidad y una apertura a un consumismo que les genera nuevas necesidades.

La región de Nangaritza tiene bastantes deficiencias en lo que se refiere a la disponibilidad de infraestructuras básicas (Ministerio de Turismo, 2013). Al mismo tiempo, la pobreza tiene una notable incidencia en la zona, de modo que, según el indice de necesidades básicas insatisfechas, se calcula que en Nangaritza la pobreza afecta al 77,4 \% de la población (Asamblea Parroquial de Zurmi, 2011, pp. 8-9).

El grupo étnico shuar posee su propia organización comunitaria, con sus órganos de gobierno tradicionales, que comparten una identidad étnica colectiva cada vez más debilitada, pero que todavía hoy cohesiona al grupo frente a las amenazas exteriores. Los shuar han mantenido enfrentamientos históricos con el Estado ecuatoriano, a la vez que las políticas de desarrollo que se han implementado en sus territorios han tenido un fuerte impacto aculturador. Durante mucho tiempo, agentes externos a las comunidades - capitaneados por el Estado, con la colaboración de los misioneros durante décadas y de ONG en tiempos más recientes- pasaron a definir la dirección del desarrollo, impulsando a partir de los años setenta y ochenta toda una serie de importantes transformaciones.

Paralelamente a este proceso, se produjo también una conflictiva colonización de sus tierras, que los ha llevado a desplazarse de forma reiterada, lo cual se ha favorecido por parte del propio Estado desde finales del siglo xIX (Rubenstein, 2005, p. 31). Esto ha derivado en diversos incidentes armados (Castillo y Carou, 2002). Ante esta continua conflictividad, en los años noventa el Estado terminó por legalizar 
unas 40.000 hectáreas de terreno colectivo para los shuar, con el fin de poder garantizarles unas tierras en las que vivir.

Por otro lado, los shuar de Nangaritza han recibido una fuerte influencia de los misioneros, quienes llegaron al territorio shuar acompañando el proceso de colonización - ya desde la época de la conquista española y hasta el siglo xx-, animados por el propio Gobierno ecuatoriano, ante su imposibilidad de controlar por la fuerza esta región (Rubenstein, 2005, p. 33). Aunque los misioneros ofrecieron a las comunidades servicios que mejoraron su calidad de vida, también impulsaron importantes transformaciones en la organización shuar que tuvieron un fuerte impacto aculturador (Rubenstein, 2005, pp. 32-39).

Actualmente, tal como ocurre en muchos otros lugares de la Amazonía, el territorio shuar se ve amenazado por los intereses de empresas extractivas (nacionales e internacionales) que desean explotar los recursos de esta región. Ello genera el riesgo de que estas empresas acaben colonizando el territorio perteneciente a las comunidades, para explotar sus recursos, con la connivencia del propio Estado, tal como ya está ocurriendo hoy en día en algunos grupos shuar de la vecina provincia de Morona Santiago. Esto ha supuesto la expulsión de la población de sus comunidades y la pérdida de sus tierras, con el empobrecimiento que de ello se deriva.

Todos estos elementos han venido a restar poder a las comunidades, lo que las ha llevado a perder buena parte de la capacidad colectiva para hacer frente a los problemas que las afectan, erosionando su propia identidad étnica.

\section{Análisis de una experiencia pionera de turismo comunitario en las poblaciones shuar del Alto Nangaritza}

La primera iniciativa turística entre los shuar de Nangaritza tuvo lugar a través del conocido Programa Podocarpus, en los años noventa. Esta iniciativa estuvo liderada por una ONG internacional de Estados Unidos, aunque también hubo participación del Estado a través del Ministerio de Turismo. La comunidad tuvo que aprobar este proyecto, pero sin haber participado en su gestación. El proyecto animó a las personas que lo desearan a poder implicarse en la actividad turística, explotando los recursos colectivos del territorio, aunque solo un sector de la población emparentado entre sí se dedicó finalmente al turismo. 
Esta iniciativa debía beneficiar inicialmente a todas las comunidades shuar del Alto Nangaritza. En realidad, se trataba de un proyecto más amplio que buscaba, entre otras cosas, la preservación del medio ambiente y la búsqueda de fuentes alternativas de ingresos - de hecho, se crearon también otros grupos comunitarios en relación con otras actividades económicas, como el grupo de la agricultura o el de la ganadería, que contaron con el aval de la comunidad-. Entre estas actividades se incluyó un proyecto de TC, con una importante inversión en equipamiento y formación. Se construyeron cabañas de alojamiento para los turistas, se realizaron acciones formativas en ámbitos diversos, como cocina e higiene, revalorización del patrimonio natural y su adecuación para el turismo, cursos de formación como guías turísticos, atención a los visitantes, recuperación de artesanías locales, entre otros, y se compraron unas embarcaciones para el transporte de los turistas. Así mismo, se equipó a la comunidad de material para la elaboración de artesanías, y cinco mujeres recibieron formación específica para ello.

Durante este tiempo se construyó un embarcadero para que la llegada de los turistas a la comunidad de Shaime en botes — único modo de acceder a la comunidad hasta ese momento- fuera más cómoda y fácil; aunque prácticamente este no ha sido utilizado, y hoy se encuentra en situación de abandono. También se llevó a algunos miembros de la comunidad a visitar otras iniciativas de TC situadas en las regiones de la costa y el oriente del país, y se les contactó con agencias de viaje ubicadas en las ciudades.

Sin embargo, pese al trabajo realizado y los recursos invertidos, esta iniciativa terminó fracasando. Las razones fueron, por una parte, el enfrentamiento de las familias por el reparto de los beneficios una vez que la ong se retiró de la zona, sin asegurar la sostenibilidad del proyecto (un hecho que potencia actualmente mucha desconfianza en la comunidad hacia cualquier otra propuesta similar, y un problema común al que se han enfrentado muchas experiencias de este tipo en Ecuador). Esta situación, como explica Cabanilla (2014, pp. 45-46), en la que las ONG han dejado los proyectos inconclusos y sin un claro plan sobre cómo se traspasará la gestión a manos de los comuneros, ha sido algo que ha minado muchos de los proyectos iniciados.

En el caso presentado, la desvinculación final de la ong tuvo lugar sin que la comunidad estuviera suficientemente preparada para ello. Así, la población no supo gestionar este proyecto en su ausencia y sin su intermediación. En la actualidad las cabañas que se construyeron ya no existen, ni tampoco las embarcaciones. De hecho, una parte de la población acusa a las dos familias de Shaime, que hoy en día se dedican al turismo de forma privada, de haberse apropiado de buena parte del 
equipamiento comunitario financiado con esta iniciativa turística. Esta situación se podría haber solventado, de alguna manera, si la población hubiera recibido una completa y adecuada capacitación. Sin embargo, en realidad únicamente asistió a algunas acciones formativas de muy corta duración.

Para autores como Timothy (2002, p. 161) o Cabanilla (2014, pp. 45-46), la escasa capacitación que las comunidades reciben para poner en marcha iniciativas turísticas es una de las grandes dificultades que encuentran, lo que a menudo lleva a que terminen fracasando. A esto se añade que no suelen contar con una asistencia técnica permanente — suele ser esporádica y desordenada-, con lo que mucha de la capacitación se ha diluido con el tiempo, sin que haya existido un seguimiento adecuado de la iniciativa.

Tampoco podemos olvidar que la comunidad aceptó sobre todo el apoyo de la ONG y del Ministerio de Turismo por su incapacidad de financiar el equipamiento y las infraestructuras necesarias para acoger a los visitantes. El problema de la dependencia económica es algo a lo que deben enfrentarse todas las comunidades (Timothy, 2002, pp. 161-162). De hecho, algunas experiencias en otros lugares se han financiado a través de empresas extractivas que, a cambio de extraer recursos de su territorio, dotaban económicamente proyectos de TC, al tiempo que facilitaban infraestructuras básicas a la comunidad. En estos casos, la distribución de los fondos también se ha convertido en fuente de conflictos para la comunidad.

No obstante, la misma comunidad sostiene que otra de las razones del fracaso de esta experiencia habría sido la falta de una organización eficaz por parte la población local y la competencia y los conflictos entre ella para beneficiarse del turismo. Como dice Carpentier (2011, p. 356), no tener estructuras de organización adecuadas también se convierte en una de las dificultades que las comunidades encuentran en el camino. Las estructuras comunitarias adquieren un papel clave en el éxito de los proyectos (Coca, 2007).

Así mismo, el hecho de que algunas de estas comunidades se encuentren muy aisladas en la selva - las más accesibles se encuentran a tres horas de viaje en automóvil desde la ciudad más próxima-, se convierte en un fuerte impedimento para el acceso de los visitantes, tal como ha ocurrido en otras experiencias similares (Timothy, 2002, p. 163).

Además, las comunidades shuar han encontrado muchos límites para publicitar su territorio como destino turístico, de modo que la comercialización del producto turístico comunitario es una de las grandes debilidades (Cabanilla, Lastra-Bravo y Pazmiño, 2017, p. 580). Incluso las mujeres que elaboran artesanías han dejado de 
hacerlo al no conocer el procedimiento para poder vender sus productos en el exterior. La comunidad se enfrenta, pues, al acceso limitado a la publicidad, al sistema de reservas y a las carencias del sistema de transporte y comunicaciones, aunque con el auge de internet este último problema ha disminuido en muchos lugares (Timothy, 2002, p. 161). Sin embargo, a día de hoy, en el Alto Nangaritza las comunidades carecen de internet, e incluso algunas comunidades tampoco cuentan con servicio eléctrico.

Las comunidades estudiadas también han encontrado problemas para el manejo de los planes de negocios y de herramientas administrativas facilitadoras de tareas como la rendición de cuentas, la formalización legal de la iniciativa de Tc, el manejo de medios electrónicos de comunicación con los visitantes o el conocimiento de la demanda potencial, problemas que son comunes a muchas otras experiencias de Tc (Cabanilla, 2014, p. 46).

Desde que finalizó la experiencia ligada al Programa Podocarpus, una familia extensa - compuesta de varios núcleos familiares — ha continuado trabajando en el ámbito turístico, pero ya no desde la perspectiva inicial, sino como negocio privado que beneficia exclusivamente a sus miembros. Pese a todo, en el caso de esta familia se sigue calificando de forma cuestionable su iniciativa como de "turismo comunitario”. Posee y gestiona el único alojamiento existente en la zona y, al mismo tiempo, es la única familia que elabora artesanía para la venta a los visitantes y cuenta con uno de los chamanes más activos de la región, que también ofrece sus servicios a los turistas. A su vez, varios miembros de la familia tienen cierta formación como guías, trabajando en el acompañamiento de grupos de turistas para visitar los atractivos naturales de la región. Aunque esta familia comenzó trabajando en el ámbito turístico a partir del Programa Podocarpus, actualmente no comparte sus beneficios con la comunidad - requisito esencial en toda experiencia de TC-, lo que ha hecho surgir el descontento en una parte de la población. No podemos olvidar que, como apunta Carpentier (2011, p. 356), la llegada de grandes cantidades de dinero y la existencia de intereses personales divergentes acaba originando fuertes conflictos internos que las comunidades muchas veces no tienen capacidad de solventar.

En este contexto, actualmente son ya varias familias de la comunidad de Shaime que también tratan de beneficiarse del turismo. Por esta razón, una familia de la misma comunidad está construyendo un nuevo alojamiento turístico. Esta misma familia participó en el anterior proyecto de TC del Programa Podocarpus, recibiendo algunas de las capacitaciones que se impartieron en este. En la actualidad también está construyendo una embarcación con la que ofrecer visitas turísticas por el río 
Nangaritza, aunque tampoco se muestra interesada en compartir sus beneficios con la comunidad.

Este surgimiento de pequeños negocios turísticos familiares y privados dentro de la comunidad está ocasionando la aparición de conflictos. Por un lado, una parte de la población acusa a ambos propietarios de haberse quedado con una parte del equipamiento que se donó a la comunidad para uso comunitario. Por otra parte, una de estas personas planea privatizar el uso de determinados espacios naturales que se encuentran dentro del lote de tierra que la comunidad le ha prestado, pretendiendo cobrar un precio a los turistas que deseen visitarlos en función de su valor paisajístico.

Este no es el único caso de personas shuar que comienzan a poner un precio a las visitas turísticas en su propiedad. Así, dos de los mayores atractivos turísticos del Alto Nangaritza (la Cueva de los Tayos y el laberinto de las Mil Ilusiones) estarían produciendo beneficios privados, algo que puede degenerar en conflictos intracomunitarios. Tal como explica Gaona (2013), los pueblos indígenas defienden la propiedad colectiva de la tierra, al entender que genera beneficios colectivos a toda la comunidad, rechazando su posible apropiación individual. En realidad, el territorio que ocupan los shuar es un lote de terreno colectivo que la propia comunidad ha repartido entre cada familia. Por esta razón, el hecho de que se construyan cercados para limitar el paso y cobrar a quienes deseen visitar los atractivos contenidos en este, generando un beneficio privado, puede llegar a poner en crisis un sistema de organización social en el que tradicionalmente los intereses colectivos constituían una prioridad. Además, como podemos observar, cada negocio turístico trata de acumular toda una serie de servicios para los visitantes, con el fin de que las ganancias familiares sean mayores, lo que genera una concentración de los beneficios turísticos en pocas manos. Finalmente, todos estos elementos contribuyen también a incrementar el proceso de monetarización de las relaciones en el seno de la comunidad.

\section{Una nueva oportunidad de desarrollo para las comunidades shuar del Alto Nangaritza a través del turismo comunitario}

Es en este contexto en el que en 2014 las comunidades shuar comenzaron a plantearse la puesta en marcha de una nueva iniciativa de Tс que superara los problemas señalados. Con el apoyo del Observatorio de Conflictos Socioambientales de la Universidad Técnica Particular de Loja (UTPL), algunos líderes comunitarios se reunieron y 
debatieron, entre otras cosas, sobre el futuro desarrollo de las comunidades, para lo cual consideraron el TC como una alternativa económica que podría mejorar el nivel de vida de la población. Sin embargo, esta iniciativa tendría que funcionar de modo paralelo a los dos negocios turísticos privados descritos ya existentes en la comunidad de Shaime.

El interés creciente de la población shuar por participar en el negocio turístico se está convirtiendo en una fuente de conflictos a medio plazo con los actuales gestores del turismo. Por una parte, la comunidad de Miazi desea poner en marcha una iniciativa de TC, tratando de evitar el actual desequilibrio entre localidades —en relación con Shaime - en lo que se refiere a la concentración de recursos turísticos. Al mismo tiempo, un grupo de mujeres de Shaime se han asociado y desean lanzar otra experiencia de $\mathrm{TC}$, lo que contribuye a generar rivalidad entre actores.

Este interés creciente por el TC surge, en buena parte, como estrategia de los shuar para dificultar la llegada de nuevas empresas extractivas a su territorio (madereras, petroleras, o mineras), tal como ha ocurrido en otras comunidades étnicas del país, en las que la población intenta justificar la preservación de zonas de especial valor ecológico (Guerrero, 2008, p. 34). A su vez, las actividades mineras están conllevando un deterioro medioambiental que resulta visible en algunas zonas, al tiempo que un incremento de la conflictividad en el seno del grupo entre quienes desean la minería y los que se oponen a esta.

La comunidad de Miazi desea poner en marcha un proyecto de TC como medio de recuperar el orgullo por su cultura ancestral, expuesta actualmente a un proceso de notable deterioro. Ya nadie viste con las ropas étnicas más que en fiestas y ante la llegada de turistas. Y los jóvenes shuar ya no hablan el idioma del grupo, pues - según uno de nuestros informantes en la comunidad- "se avergüenzan de ser shuar”. Así mismo, cada vez más los intereses comunitarios son relegados ante un individualismo creciente. Sin embargo, muchos de ellos ven en el TC una oportunidad para fortalecer una identidad étnica que hoy en día está en crisis, o se muestran interesados en poner en marcha un museo participativo de la identidad shuar.

No obstante, el TC conlleva también impactos culturales no deseados por la población, que erosionan la cultura tradicional del grupo y deterioran la organización comunitaria. Por ejemplo, existen determinadas prácticas rituales y simbólicas de los shuar que actualmente se están ofreciendo a los visitantes, que antes fomentaban la cohesión del grupo y que ahora pierden parte de su sentido en un contexto turístico (como la fiesta de la txonta y la de la culebra, o la ceremonia de la ayahuasca, y otros rituales que se celebraban para agradecer las cosechas, prevenir picaduras o para 
visionar el futuro de la comunidad). Este nuevo uso de los rituales del grupo estaría generando pequeños conflictos con las familias que no se dedican al turismo, las cuales no ven con buenos ojos que los visitantes deambulen por el espacio comunitario, así como que se usen sus rituales sagrados con fines económicos (Cabanilla, 2015).

Sin embargo, paradójicamente, la actividad turística estaría animando también a la población local a mantener una parte de su patrimonio. Así, los shuar están recuperando una artesanía que no elaboraban desde hace décadas. También, como consecuencia del turismo, la población — que hace más de una década que abandonó el uso de su vestimenta étnica- cambia sus ropas occidentales por su vestimenta tradicional ante la llegada de los visitantes. De modo que, como explican Gascón y Cañada (2005, p. 78), el desarrollo turístico estaría contribuyendo a estimular el interés de los residentes por sus propias manifestaciones culturales que, de otro modo, desaparecerían, aunque de forma y con funciones diferentes.

Por otra parte, para poder poner en marcha nuevas iniciativas de TC las comunidades shuar encuentran numerosas e importantes dificultades. El primer elemento de esa cadena de obstáculos es el financiero. Poner en marcha infraestructuras turísticas y capacitarse para poder realizar una correcta gestión turística hace necesario un elevado capital económico del que las comunidades shuar no disponen. En nuestro caso de estudio, son varias las instancias regionales que han denegado su apoyo económico al proyecto, como la Municipalidad de Nangaritza y la Asamblea Parroquial de Zurmi; mientras que otras se comprometieron a apoyar este proyecto, pero sin que hasta el momento se haya materializado, como ocurre con el Gobierno Provincial de Zamora. En cuanto a la financiación del Ministerio de Turismo, se convierte en una empresa casi imposible de solventar ante la enorme gestión burocrática necesaria para ello. Respecto a las ONG, estas apoyan financieramente a las comunidades en otros ámbitos relacionados con el desarrollo, pero de momento no en el del TC. La propia UTPL es la única entidad que ha financiado una parte de la formación inicial necesaria, pero que sin duda resulta absolutamente insuficiente.

Otra de las limitaciones que la población shuar debe solventar para poner en marcha una iniciativa de TC son los prejuicios que la sociedad mestiza tiene sobre ellos. Los grupos indígenas de Ecuador — como ha ocurrido en otros lugares del mundo- han sido social, económica y políticamente excluidos (Contreras, 2000). Aún hoy, buena parte de la sociedad mestiza tiene una imagen muy estereotipada de la población indígena. El presidente de la Municipalidad de Nangaritza reflejaba estos prejuicios al negarse a aportar financiación al proyecto de TC impulsado por la UTPL, argumentando que los shuar son “derrochadores por naturaleza y poco dados 
al trabajo", tal como nos expresó durante nuestra estancia en la zona. Lo mismo ocurría con el presidente de la Asamblea Parroquial de Zurmi, que negó el valor de las aportaciones culturales y económicas de los indígenas, un fenómeno muy extendido entre la sociedad mestiza.

Igualmente, los shuar deben competir con agentes turísticos foráneos, considerablemente mejor preparados y que gozan de un apoyo financiero y logístico por parte de la administración mucho mayor. En Nangaritza, lindando los límites shuar, existen desde hace muchos años unas cabañas turísticas que son propiedad de una persona mestiza venida de la ciudad, y que organiza visitas a los atractivos turísticos naturales ubicados en territorio de los shuar. Algunos líderes shuar consideraban que esta persona "está saqueando las comunidades", como nos manifestaron durante nuestra estancia. Además, actualmente son varias las personas de la comunidad mestiza vecina de Las Orquídeas interesadas en beneficiarse del turismo, visitando con los turistas los recursos pertenecientes a las comunidades.

Conscientes de que personas venidas de fuera de su territorio tratan de explotar recursos turísticos que consideran que les pertenecen, en ocasiones los shuar se han organizado para expulsar a los forasteros. En este sentido, la comunidad ha logrado paralizar varios proyectos turísticos impulsados por personas ajenas a la comunidad, en los que no se ha realizado una consulta previa a las comunidades - como el de Ciudad Perdida ${ }^{3}$ - . Al mismo tiempo, en la actualidad el Ministerio de Turismo estaría implementando un Plan Estratégico de Desarrollo Turístico para el Alto Nangaritza, sin consulta a las comunidades, y cuyo beneficio alcanzaría también a personas mestizas venidas desde otros lugares, lo que probablemente se convierta en otra fuente de conflicto.

\section{Conclusiones}

El TC se ha convertido en muchas comunidades indígenas en una importante alternativa económica, que en ocasiones ha mejorado el nivel de vida de las poblaciones, ha reforzado la cultura y la organización comunitaria y ha limitado otro tipo de actividades económicas bastante nocivas para el medio natural privilegiado en el que frecuentemente se desarrollan.

3 Lugar ubicado en territorio shuar, donde existen restos arqueológicos de una ciudad fundada por los españoles durante los siglos XVI y XVII. 
Sin embargo, ello no siempre es así. Cuando el Tc llega a determinados contextos como el de los shuar, que llevan tiempo afectados por procesos de desestructuración comunitaria que les impiden dar respuestas colectivas a las necesidades y retos que se les presentan, puede acabar convirtiéndose en una fuente de conflictos por el reparto de los beneficios, por la explotación del territorio o por el uso de los rituales, acabando por ahondar en la fragmentación comunitaria. En el caso analizado es visible la creciente división interna de intereses en torno al turismo, con el desarrollo de negocios turísticos privados en el seno de la comunidad, y cómo esta se ha mostrado incapaz hasta el momento de poner en marcha una iniciativa exitosa de TC.

En buena parte de los casos, las experiencias de TC son apoyadas por entidades externas que muchas veces conocen de un modo superficial la situación real de las poblaciones en que se implantan los proyectos. Los pueblos indígenas han sufrido durante siglos un proceso de exclusión y discriminación en el seno de sus Estados que no puede obviarse. Buena parte de sus tierras han sido colonizadas, y por ello se han visto obligados a sedentarizarse, lo que los lleva a adaptarse a un nuevo modo de vida que no han decidido. En este sentido, las ONG y otras entidades financiadoras tratan de ayudar a las comunidades, a través de limitadas intervenciones y de la puesta en marcha de proyectos que en muchas ocasiones no se adaptan a las necesidades del entorno o que no tienen en cuenta la voluntad de la población local, con lo que consecuentemente terminan fracasando.

El TC representa una oportunidad para las comunidades, al implicar un mayor control de su proceso de desarrollo. Pero para que esta actividad pueda resultar empoderadora es necesario — tal como nos transmitía el presidente de la Feptcetrabajar en una primera fase un fortalecimiento de las estructuras comunitarias y lanzar un proceso de reflexión colectiva en el grupo sobre las implicaciones y riesgos que el TC suele conllevar, con el fin de poder prevenir dichos impactos. Sin embargo, no siempre las entidades que apoyan este tipo de iniciativas están preparadas para ello. Frecuentemente las instituciones tienen rígidos plazos establecidos que deben cumplirse, lo que impide que se respeten los ritmos necesarios de las comunidades para decidir y organizarse de forma adecuada. También ocurre a menudo que los actores externos abandonan los proyectos sin que las comunidades estén suficientemente preparadas para asegurar su continuidad.

Pese a todo, nos atrevemos a afirmar que el TC puede representar una importante herramienta de desarrollo para los grupos indígenas. En todo caso, es mucho menos nocivo que otras modalidades de desarrollo turístico - y que otras actividades económicas - que son decididas y gestionadas por actores externos a las comunidades, y 
que acaban beneficiando marginalmente a las poblaciones locales. Las ONG y las agencias gubernamentales deberían flexibilizar los tiempos necesarios para desarrollar este tipo de iniciativas, respetando los ritmos y las necesidades de las propias comunidades. Así mismo, deberían realizarse estudios de diagnóstico participativo previos que permitan un conocimiento en profundidad de las peculiaridades del contexto en que se va a intervenir, garantizando beneficios colectivos. Solo así el TC conducirá a las poblaciones indígenas hacia un desarrollo realmente empoderador.

\section{Sobre las autoras}

María Jesús Berlanga Adell. Licenciada en Antropología Social y Cultural por la Universitat Rovira i Virgili, doctora en Cooperación al Desarrollo y diplomada universitaria en Trabajo Social por la Universidad de Valencia. Actualmente es profesora doctora en la Universidad Católica de Valencia. Sus principales líneas de investigación son el turismo, el desarrollo social, las migraciones y las identidades. Sus publicaciones en revistas figuran en la Revista Internacional de Estudios del Desarrollo, Migraciones Internacionales, Revista Andaluza de Antropología, Pasos o Arxius de Ciències Socials. A lo largo de estos años ha llevado a cabo investigaciones, y ha colaborado en proyectos implementados tanto en Chile y Ecuador como en Marruecos y España. También ha participado en numerosas formaciones especializadas y de posgrado en diferentes universidades españolas, de Chile y Ecuador, donde ha venido impartiendo de manera regular seminarios de formación en investigación para docentes de la Universidad Técnica Particular de Loja.

Maritza Elizabeth Ochoa Ochoa. Maestra en Derecho por la Universidad Nacional Autónoma de México, magíster en Desarrollo Comunitario por la Universidad Nacional de Loja y abogada de la República del Ecuador. Es investigadora del Departamento de Ciencias Jurídicas de la Universidad Técnica Particular de Loja y docente de la carrera de Derecho y de la Maestría en Derecho Penal. Mención Derecho Procesal Penal en modalidad presencial abierta y a distancia. Es miembro del grupo de investigación "Estudios sobre Constitucionalismo Latinoamericano y Derechos Humanos”. Las líneas de investigación que ha desarrollado se enfocan en el estudio de derechos constitucionales y fundamentales de los grupos de atención prioritaria. 


\section{Referencias}

Asamblea Parroquial de Zurmi. (2011). Plan de Desarrollo y Ordenamiento Territorial del cantón Nangaritza. Inédito.

Blackstock, K. (2005). A critical look at community based tourism. Community Development Journal, 4O(1), 39-49. DoI: https://doi.org/10.1093/cdj/bsi005

Brohman, J. (1996). New directions in tourism for third world development. Annals of Tourism Research, 23(1), 48-70. DoI: https://doi.org/10.1016/0160-7383(95)00043-7

Cabanilla, E. (2014). El turismo comunitario en Ecuador, bajo el paradigma de la complejidad y el Sumak Kawsay. Bio Scriba, 7(1), 30-49.

Cabanilla, E. (2015). Impactos culturales del turismo comunitario en Ecuador sobre el rol del chamán y los ritos mágico-religiosos. Estudios y Perspectivas en Turismo, 24(2), 356-373.

Cabanilla, E., Lastra-Bravo, X. y Pazmiño, J. (2017). El turismo doméstico ¿Demanda potencial de los centros de turismo comunitario en Ecuador? Pasos, 15(3), 579-590. DoI: https://doi. $\operatorname{org} / 10.25145 / \mathrm{j}$.pasos.2017.15.039

Carpentier, J. (2011). Tourisme communitaire, conflits internes et développement local. Butlletin de l'Institut Français d'Études Andines, 40(2), 349-373. DoI: https://doi.org/10.4000/ bifea.1503

Castillo, L. y Carou, H. C. (2002). Reinvención de la identidad étnica, nuevas territorialidades y redes globales: el Estado multi y pluricultural en Colombia y Ecuador. Sociedad y Economía, 3, 55-76.

Chiriboga, M. y Wallis, B. (2010). Diagnóstico de la pobreza rural en Ecuador y respuestas de política pública. Centro Latinoamericano para el Desarrollo Rural. Recuperado de https:// www.rimisp.org/wp-content/files_mf/1366317392Diagnosti...pdf

Coca, A. (2007). Machacuyacu, turismo y organización comunitaria. En E. Ruiz y D. Solís (Coord.), Turismo comunitario en Ecuador: desarrollo y sostenibilidad social (pp. 21-40). Quito: Abya-Yala.

Contreras, M. Á. (2000). Los indígenas y la globalización en América Latina. Derechos Humanos. Órgano Informativo de la Comisión de Derechos Humanos del Estado de México, 44, 137 149. Recuperado de http://historico.juridicas.unam.mx/publica/librev/rev/derhum/ cont/44/pr/pr26.pdf

Feptce. (2007). Manual de calidad para la gestión del turismo comunitario del Ecuador. Quito, Ecuador: Editorial Feptce.

Gaona, G. (2013). El derecho a la tierra y protección del medio ambiente por los pueblos indígenas. Nueva Antropología. Revista de Ciencias Sociales, 78(26), 141-161. Recuperado de https://dialnet.unirioja.es/servlet/articulo?codigo $=5302975$

García-Andreu, H. y Ullán, J. (2019). ¿Todavía existe la comunidad?: turismo comunitario y nuevas formas de organización social a caballo entre la tradición y la modernidad. Obets. Revista de Ciencias Sociales, 14(1), 119-143. Dor: 10.14198/OBETS2019.14.1.04 
El turismo comunitario como herramienta de desarrollo de los pueblos indígenas...

García, C. (2016). Turismo comunitario en Ecuador: ¿Quo vadis? Estudios y Perspectivas en Turismo, 25, 597-614.

Gascón, J. y Cañada, E. (2005). Viajar a todo tren: turismo, desarrollo y sostenibilidad. Barcelona: Icaria.

Guerrero, P. (2008). Notas para una guía teórica y metodológica sobre cultura, identidad, interculturalidad, patrimonio y turismo comunitario. Quito, Ecuador: Editorial Feptce.

Instituto Nacional de Estadística y Censos (INEC). (2010). Censo de Población y Vivienda, Gobierno de la República del Ecuador.

Maldonado, C. (2005). Pautas metodológicas para el análisis de experiencias de turismo comunitario. Ginebra, Suiza: OIT. Recuperado de http://oit.org/wcmsp5/groups/public/---ed_ emp/---emp_ent/---fp_seed/documents/publication/wcms_117525.pdf

Ministerio de Turismo de Ecuador. (2010). Reglamento para los Centros Turísticos Comunitarios, Acuerdo Ministerial 16. Registro Oficial 154 de 19 de marzo. Quito.

Ministerio de Turismo de Ecuador. (2013). Proyecto de desarrollo del circuito turístico del Alto Nangaritza. Quito: inédito.

Rubenstein, S. (2005). La conversión de los shuar. Iconos. Revista de Ciencias Sociales, 22, 27-48. DoI: https://doi.org/10.17141/iconos.22.2005.99

Ruiz, E. y Solís, D. (Coord.) (2017). Turismo comunitario en Ecuador: desarrollo y sostenibilidad social. Quito: Abya-Yala.

Ruiz, E., Hernández, M., Coca, A., Cantero, P., y Del Campo, A. (2008). Turismo comunitario en Ecuador. Comprendiendo el Community-Based Tourism desde la comunidad. Pasos, 6(3), 399-418. Recuperado de http://pasosonline.org/Publicados/6308/PS0308_2.pdf

Solís, D. (2007). De la resistencia a la sostenibilidad. El proceso histórico del turismo comunitario en Ecuador y sus retos actuales. En E. Ruiz y D. Solís (Coords.), Turismo comunitario en Ecuador: desarrollo y sostenibilidad social (pp. 21-40). Quito: Abya-Yala.

Timothy, D. (2002). Tourism and Community Development Issues. En R. Sharpley y D. Telfer (Eds.), Tourism and development. Concepts and issues (pp. 149-164). Bristol, Reino Unido: Channel View Publications.

Tosun, C. (2000). Limits to community participation in the tourism development process in developing countries. Tourism Management, 21(6), 613-633. DoI: https://doi.org/10.1016/ S0261-5177(00)00009-1

Unesco. (1994). La dimension culturelle du développement. París: Unesco. Recuperado de http:// unesdoc.unesco.org/images/0017/001777/177716fo.pdf

Wikimedia Commons. (2019a). Ecuador. Recuperado de https://upload.wikimedia.org/wikipedia/commons/9/9a/Ecuador\%2C_administrative_divisions_-_es_-_colored.svg

Wikimedia Commons. (2019b). Cantones de Zamora Chinchipe. Recuperado de https://upload. wikimedia.org/wikipedia/commons/thumb/b/bo/Cantones_de_Zamora_Chinchipe. png/250px-Cantones_de_Zamora_Chinchipe.png 
MARÍA JESÚS BERLANGA ADELL Y MARITZA ELIZABETH OCHOA OCHOA

World Wide Foundation International. (2001). Guidelines for community-based ecotourism development. Suiza. Recuperado de https://d2ouvy59p0dg6k.cloudfront.net/downloads/ guidelinesen.pdf 